\title{
ESTIMATION OF STEADY STATE PROBABILITY DISTRIBUTION OF SYSTEM SIZE IN M/M/1 QUEUE
}

\author{
Jayakrishna Udupa $H$.
}

\begin{abstract}
Recently Choudhury and Borthakur (2008) obtained classical and Bayesian estimators of performance measures based on a random sample of size $n$ from a geometric distribution with mean $\rho /(1-\rho)$, which is the steady state probability distribution of system size. Here we obtain classical estimators, the Maximum Likelihood (ML) and Uniformly Minimum Variance Unbiased (UMVU) estimators, and Bayesian estimators of $P_{k}, k=0,1,2, \ldots$ relative to beta prior distribution and Weighted Squared Error Loss (WSEL) function as well as relative to Standard Two-Sided Power (STSP) distribution and squared error loss / WSEL functions. Bayes, ML and UMVU estimates of the probability that the server is idle are also computed. Also Consistent Asymptotic Normality (CAN) for $P_{k}, k=0,1,2, \ldots$ are examined.
\end{abstract}

\section{Introduction}

Problems of estimation have been the interest of queuing researchers since the mid 1950's. Early researchers in classical estimation problems of queuing parameters

- Department of Statistics, Christ University, Hosur Road, Bangalore - 560029, Phone: 080 - 40129335, Email: jayakrishna.udupa@christuniversity.edu 
include Clarke (1957), Wolf (1965) and Cox (1965) while that for Bayesian estimation problems include Muddapur (1972), Armero (1985) and Basawa et al. (1981). In the past few decades there has been a surge in research related to Bayesian inference for queuing parameters. Research in the last decade includes Armero and Conesa $(1998,2000)$, Conti (1998), Armero and Bayarri(1999), Butler and Huzurbazaar (2000), Zheng and Seila (2000), and Keissler and Lund (2009). Estimation in M/M/l queve was studied by Srinivas et al. (2009) using the Imbedded Markov Chain (IMC) analysis of $M / G / 1$ queve. They obtained Maximum Likelihood (ML) and Uniformly Minimum Variance Unbiased (UMVU) estimators of steady state measures of system performance. Sharma and Kumar (1999) obtained ML estimators of the same measures as well as Bayesian estimators relative to Squared Error Loss (SEL) function and beta prior distribution based on a sample of size K, from $K$ independent $M / M / 1$ queves, from geometric distribution

$$
p(x)=(1-\rho) \rho^{x}, \quad x=0,1,2, \ldots
$$

which is the steady state distribution of system size and is denoted by $p_{x}$. Recently Choudhury and Borthakur (2008) derived the Bayes estimators of the traffic intensity parameter relative to SEL and beta / truncated uniform priors, based on a random sample from (1.1) using the ergodicity property of IMC to argue that the sample observations are independent. We assume throughout that there exists a random sample of observations $\underset{\sim}{X}=X_{1}, X_{2}, \ldots, X_{n}$ on $X$ with geometric p.m. $f$ in (1.1).

In this paper we intend to obtain the classical and Bayesian estimators of the steady state probability distribution of system size given by

$$
p_{k}=(1-\rho) \rho^{k}, k=0,1,2, \ldots
$$

which is the same as the distribution in (1.1). The ML and UMVU estimators are derived in section 2 and 3 respectively. Section 4 deals with the CAN for $p_{k}$. Bayesian estimators of $p_{k}$ relative to beta prior and loss functions SEL and Weighted Squared Error Loss (WSEL) are derived in section 5. In contrast, in section 6, the Bayes estimators of $p_{k}$ relative to Standard Two-Sided Power (STSP) distribution and SEL / WSEL functions are obtained.

\section{Maximum Likelihood Estimation}

The likelihood of the observed sample drawn from (1.1) is given by

$$
L(\rho ; \underset{x}{x})=(1-\rho)^{n} \rho^{n \bar{x}}
$$


Now differentiating the log of the likelihood function with respect to $\rho$ and equating it to zero gives the MLE of $\rho$ as

$$
\hat{\rho}=\frac{\bar{x}}{\bar{x}+1}
$$

By using the invariance property of MLE, we thus obtain the MLE of steady state probability distribution as

$$
\hat{p}_{k}=\left(1-\frac{\vec{x}}{\bar{X}+1}\right)\left(\frac{\vec{x}}{\bar{x}+1}\right)^{k}, k=0,1,2, \ldots
$$

\section{Best Unbiased Estimation}

The distribution in (1.1) is a member of the Power Series family of distributions (P.S.D) with p.m.f

$$
f(x \mid \theta)=\frac{a(x) \theta^{x}}{c(\theta)}, \quad x \in T^{\prime}
$$

where $T$ ' is the support of $X$. Further $T=\sum_{i}^{n} X_{i}$ is a Complete Sufficient Statistic (C.S.S) for P.S.D. It is well known (See Lehmann (1983, P. 96 and Singh (1979)) that UMVU estimator of probabilities of P.S.D based on a sample of size $n$ from P.S.D is given by

$$
\tilde{f}(x \mid \theta)=\frac{a(x) A(t-x, n-1)}{A(t, n)}, x \in T^{\prime},
$$

where $A(t, n)$ is the coefficient of $\dot{\theta}^{\prime}$ in the expansion of $\{c(\theta)\}^{n}$. Using this for our case of estimation of geometric probabilities $p_{k}$ based on a sample of size $n$ from (1.1), we have the UMVU estimators of steady state probabilities given by

$$
\tilde{p}_{k}=\frac{\left(\begin{array}{c}
n+t-k-2 \\
t-k
\end{array}\right)}{\left(\begin{array}{c}
n+t-1 \\
t
\end{array}\right)}, t \geq k(\geq 0), n>1
$$


as $a(x)=1$ and $A(t, n)=\left(\begin{array}{c}n+t-1 \\ t\end{array}\right)$

\section{CAN Property}

The Consistent Asymptotic Normality (CAN) property is examined for $p_{k}$ following Kale (2005). To accomplish this we first obtain the consistent estimators. Now using the method of moments we can obtain the consistent estimator of $\rho$ as the solution of moment equation $\bar{X}_{n}=\frac{\rho}{1-\rho}$. For this the condition $\frac{d \mu}{d \rho}=\frac{1}{(1-\rho)^{2}} \neq 0$ and is continuous for $\rho \in(0,1)$ is satisfied, which is sufficient for $\mu^{-1}$ to exist. The solution of moment equation is $\rho^{*}=\frac{\bar{X}_{n}}{\bar{X}_{n}+1}$ and is a consistent estimator of $\rho$. Further, to obtain CAN estimator of $L=\frac{\rho}{1-\rho}$ we note that by Central Limit Theorem (CLT)

$$
\bar{X}_{n}-A N\left(L, \frac{L}{n(1-\rho)}\right)
$$

Thus, by definition, $\bar{X}_{n}$ is CAN for $L$ as $\bar{X}_{n} \stackrel{P}{\longrightarrow} L$ by Weak law of Large Numbers (WLLN). As $\rho=\frac{L}{L+1}$ and $\frac{d g(L)}{d L} \neq 0$ and is continuous, by invariance property of CAN estimators, $\frac{\bar{X}_{n}}{\bar{X}_{n}+1}$ is CAN for $\rho$, with

$$
\frac{\bar{X}_{n}}{\bar{X}_{n}+1} \sim A N\left(\rho, \frac{L}{n(1-\rho)}\left(\frac{1}{L+1}\right)^{4}\right)
$$

As $p_{k}=(1-\rho) \rho^{k}=g(\rho)$ and $\frac{d g(\rho)}{d \rho} \neq 0$ and is continuous, by invariance property of CAN estimators

$$
\left(1-\frac{\bar{X}_{n}}{\bar{X}_{n}+1}\right)\left(\frac{\bar{X}_{n}}{\bar{X}_{n}+1}\right)^{k}
$$


is CAN for $(1-\rho) \rho^{k}$ with

$$
\left(1-\frac{\bar{X}_{n}}{\bar{X}_{n}+1}\right)\left(\frac{\bar{X}_{n}}{\bar{X}_{n}+1}\right)^{k} \sim \operatorname{AN}\left((1-\rho) \rho^{k}, \frac{\rho^{k}[k(1-\rho)-\rho]}{n(1-\rho)^{2}}\right) .
$$

\section{Bayes Estimation Relative to Beta Prior}

The parametric space of $p_{k}$ is $\Theta=\left\{p_{k}: 0<p_{k}<1, k=0,1,2, \ldots\right\}$.The Bayes estimators of $p_{k}$ relative to $S E L$ function given by

$$
L\left(\hat{p}_{k}, p_{k}\right)=\left(\hat{p}_{k}-p_{k}\right)^{2}, \quad k=0,1,2, \ldots
$$

where $\hat{p}_{k}$ is an estimate of $p_{k^{\prime}}$ and beta prior distribution for $\rho$, denoted by $\mathrm{Be}_{1}(\alpha, \beta)$, given by

$$
\pi(\rho \mid \alpha, \beta)=\frac{1}{B(\alpha, \beta)} \rho^{\alpha-1}(1-\rho)^{\beta-1}, \quad 0<\rho<1,
$$

with the parameter space of the prior parameters being $\{(\alpha, \beta): \alpha \geq 0, \beta \geq 0\}$, can be easily derived and turns out to be

$$
\hat{p}_{k_{(B)}}=\frac{B(n \bar{X}+\alpha+k, n+\beta+1)}{B(n \bar{X}+\alpha, n+\beta)}, k=0,1,2, \ldots
$$

The Bayes estimator of $p_{k}$ relative to WSEL function given by

$$
L\left(\hat{p}_{k}, p_{k}\right)=w(\rho)\left(\hat{p}_{k}-p_{k}\right)^{2}, k=0,1,2, \ldots
$$

and prior (5.2) turns out to be

$$
\hat{p}_{k(B)}=\frac{B(n \bar{X}+\alpha+k-1, n+\beta+3)}{B(n \bar{X}+\alpha-1, n+\beta+2)}, \quad k=0,1,2, \ldots
$$

where the weight function $w(\rho)=\frac{(1-\rho)^{2}}{\rho}$. 
A random sample of size 10 was simulated 10000 times from the distribution (1.1) and the Bayes estimates of $p_{0}$ (probability that the server is idle) relative to beta prior distribution $(\alpha=2, \beta=5)(5.2)$ and loss function (5.3) were obtained and is given in the following table.

Table 1. Bayes (WSEL), ML, UMVU estimates of $P_{0}$

\begin{tabular}{|c|c|c|c|}
\hline$\rho$ & MLE & UMVUE & $\hat{p}_{\mathrm{O}_{\{\beta \mid}}$ \\
\hline 0.19 & 0.81103 & 0.794351 & 0.797 \\
\hline 0.39 & 0.610128 & 0.584795 & 0.669555 \\
\hline 0.59 & 0.409668 & 0.384451 & 0.50883 \\
\hline 0.79 & 0.210261 & 0.193299 & 0.300566 \\
\hline 0.99 & 0.0099914 & 0.0090013 & 0.016834 \\
\hline
\end{tabular}

\section{Bayes Estimation Relative to STSP Prior}

The STSP distribution was introduced by Van Dorp and Kotz (2002) as a distribution which models the difference between maturity interest rates of two successive time periods (month). This distribution is used as the prior distribution of $\rho$ and is given by

$$
\pi(\rho \mid \theta, \alpha)=\left\{\begin{array}{cl}
\alpha\left(\frac{\rho}{\theta}\right)^{\alpha-1}, & 0<\rho \leq \theta \\
\alpha\left(\frac{1-\rho}{1-\theta}\right)^{\alpha-1}, & \theta \leq \rho<1
\end{array}\right.
$$

The posterior distribution of $\rho$, given the data, is given by

$$
\pi(\rho \mid \theta, \alpha)= \begin{cases}\frac{\rho^{n \bar{x}+\alpha-1}(1-\rho)^{n}}{\int_{0}^{\theta} \rho^{n \bar{n}+\alpha-1}(1-\rho)^{n}}, & 0<\rho \leq \theta \\ \frac{\rho^{n \bar{x}}(1-\rho)^{n+\alpha-1}}{\int_{0}^{\theta} \rho^{n \bar{x}}(1-\rho)^{n+\alpha-1}}, & \theta<\rho \leq 1\end{cases}
$$


Thus the Bayes estimator of $p_{k^{\prime}}$ relative to Squared Error Loss (SEL) in (5.1), is given by

$$
\hat{p}_{k_{(B)}}= \begin{cases}\frac{B(0, \theta, n \bar{X}+\alpha+k, n+2)}{B(0, \theta, n \bar{X}+\alpha, n+1)}, & 0<\rho \leq \theta \\ \frac{B(\theta, 1, n \bar{X}+k+1, n+\alpha+1)}{B(\theta, 1, n \bar{X}+1, n+\alpha)}, & \theta \leq \rho<1\end{cases}
$$

for $k=0,1,2, \ldots$ and $B(p, q, r, s)$ is the generalized incomplete beta function $\operatorname{defined~by} \int_{p}^{q} \rho^{r-1}(1-\rho)^{s-1} d \rho$

The Bayes estimator of $p_{k^{\prime}}$ relative to WSEL function (5.3) and STSP prior in (6.1), is given by

$$
\hat{p}_{k_{(B)}}= \begin{cases}\frac{B(0, \theta, n \bar{X}+\alpha+k-1, n+4)}{B(0, \theta, n \bar{X}+\alpha-1, n+3)}, & 0<\rho \leq \theta \\ \frac{B(\theta, 1, n \bar{X}+k, n+\alpha+3)}{B(\theta, 1, n \bar{X}, n+\alpha+2)}, & \theta \leq \rho<1\end{cases}
$$

A random sample of size 10 was simulated 10000 times from the distribution (1.1) and the Bayes estimates of $p_{0}$ (probability that the server is idle) relative to STSP prior distribution (6.1) and loss function (5.3) was obtained and is presented in the following table.

Table 2. Bayes (WSEL), ML, UMVU estimates of $P_{0}$

\begin{tabular}{|c|c|c|c|c|c|}
\hline$\theta / \rho$ & 0.19 & 0.39 & 0.59 & 0.79 & 0.99 \\
\hline 0.1 & 0.812544 & 0.685398 & 0.492784 & 0.271528 & 0.0139323 \\
\hline 0.2 & 0.868666 & 0.664285 & 0.492735 & 0.271528 & 0.0139323 \\
\hline 0.3 & 0.827506 & 0.610774 & 0.490143 & 0.271528 & 0.0139323 \\
\hline 0.4 & 0.805688 & 0.698192 & 0.470981 & 0.271528 & 0.0139323 \\
\hline 0.5 & 0.797908 & 0.658887 & 0.423153 & 0.271436 & 0.0139323 \\
\hline 0.6 & 0.796264 & 0.641683 & 0.499428 & 0.267626 & 0.0139323 \\
\hline 0.7 & 0.796088 & 0.637869 & 0.4657 & 0.239382 & 0.0139323 \\
\hline
\end{tabular}


Table 2. Contd.

\begin{tabular}{|c|c|c|c|c|c|}
\hline$\theta / \rho$ & 0.19 & 0.39 & 0.59 & 0.79 & 0.99 \\
\hline 0.8 & 0.796081 & 0.637572 & 0.457899 & 0.27189 & 0.0139323 \\
\hline 0.9 & 0.796081 & 0.637567 & 0.457586 & 0.252315 & 0.0139323 \\
\hline MLE & 0.81103 & 0.610128 & 0.409668 & 0.210261 & 0.00999141 \\
UMVUE & 0.794351 & 0.584795 & 0.384451 & 0.193299 & 0.0090013 \\
\hline
\end{tabular}

\section{Conclusion}

The Bayes, ML and UMVU estimators of $P_{k}$ were obtained and computed for $p_{0}$ following the research in Srinivas and Jayakrishna (2009), where the interest was in expected system size. We are aware of the fact that computations do not reveal about the performance of estimators. Also Bayes estimation was restricted to SEL and WSEL loss functions. However, Bayes estimation relative to other loss functions as well as comparison of estimators is under consideration and may possibly be part of another communication.

\section{Acknowledgment}

I am thankful to my research guide Dr. V. Srinivas, Department of Statistics, Bangalore University for his guidance.

\section{References:}

1. Armero $C$ (1985) Bayesian analysis of $M / M / 1 / \infty / F I F O$ queves, In: Bayesian Statistics 2 (ed. By J.M. Bernardo, M.H. Degroot, D. V. Lindley, and A.F.M Smith) 613-617

2. Armero C, Conesa D (1998) Inference and prediction in bulk arrival queves and queves with service in stages. Appl: Stochastic Models and Data Anal 15:35-46

3. Armero C, Conesa D (2000) Prediction in Markovion bulk arrival queves. Queving Syst $34: 327.350$

4. Armero C, Bayarri MJ (1999) Dealing with uncertainties in queves and networks of queves: A Bayesian approach. In: Ghosh S (ed.) Multivariate analysis, design and survey sampling. Marcel Dekker, New York, pp 579.608

5. Basawa IV, Prabhu NU (1981) Estimation in single senver queues. Nov. Res. Log. Quart. 28: $475-487$ 
6. Butler RW, Huzurbazaar AV (2000) Bayesian prediction of waiting times in stochastic models. Canad J Statist 28:311.325

7. Choudhury A, Borthakur $A C$ (2008) Bayesian inference and prediction in the single server Markovian queue Metrika 67:371-383 8. Clarke $A B$ (1957) Maximum likelihood estimates in a simple queve. Ann. Math. Stats. 28:
$1036-1040$

9. Conti PL (1998) Large sample Boyesian analysis for Geo/G/1 discrete time queuing models. Technical report, Dipartmento di Statistica, Probabilitia e Statistiche Applicate, Universita di Roma "La Sapienza".

10. Cox DR (1965) Some problems of statistical analysis connected with congestion, In: Proc. Of the SYMP. on Congestion theory (ed. By W.L. Smith and W.B. Wilkinson), University of North Carolina at Chapel Hill, NC

11. Kale BK (2005) A First Course on Parametric Inference. Narosa Publishing House Pvt.ltd.

12. Kiessler PC, Lund R (2009) Technical note: Traffic intensity estimation. Ncvol Res Logistics $56: 385-387$

13. Lehmonn EL (1983) Theory of Point Estimation, John Wiley, New York

14. Muddapur MV (1972) Bayesian estimates of parameters in some queuing models. Ann. Inst. Stat. Math 24:327.331

15. Sharma KK, Kumar V (1999) Inference on $M / M / 1:(\infty$ : FIFO) queve systems. Opsearch 36: $26 \cdot 34$

16. Singh $\mathrm{J}(1980)$ Minimum variance unbiased estimation of probability densities. Aust. Jour. Stat. 22 328-331

17. Srinivas V, Jayakrishna Udupa H (2009) Bayesian and classical estimbtion in steady stcte $M / M / 1$ queve. Sent for publication.

18. Srinivas V, Subba Rao S, Kale BK (1997) Estimation of measures in $M / M / 1$ queve. Sent for publication.

19. Van Dorp JR, Kotz S (2002) The two-sided power distribution and its properties: With applications in financial engineering. The Amer. Stat. 56(2): 90-99

20. Wolff RW (1965) Problems of statistical inference for birth-and-death queving models. Oper. Res. 13:343-357 21. Zheng S, Seila AF (2000) Some well-behaved estimators for the $M / M / 1$ queve. Oper. Res.
Lett. 26: 231-235 